\title{
Methods to electrophoretically stretch DNA: microcontractions, gels, and hybrid gel-microcontraction devices
}

\author{
Greg C. Randall, ${ }^{a}$ Kelly M. Schultz ${ }^{b}$ and Patrick S. Doyle*a \\ Received 28th October 2005, Accepted 14th February 2006 \\ First published as an Advance Article on the web 7th March 2006 \\ DOI: 10.1039/b515326c
}

The ability to controllably and continuously stretch large DNA molecules in a microfluidic format is important for gene mapping technologies such as Direct Linear Analysis (DLA). We have recently shown that electric field gradients can be readily generated in a microfluidic device and the resulting field is purely elongational. We present a single molecule fluorescence microscopy analysis of T4 DNA (169 kbp) stretching in the electric field gradients in a hyperbolic contraction microchannel. In addition, we are able to selectively pattern a crosslinked gel anywhere inside the microchannel. With an applied electric field, DNA molecules are forced to reptate through the gel and they moderately stretch as they exit the gel. By placing a gel immediately in front of the hyperbolic contraction, we bypass "molecular individualism" and achieve highly uniform and complete stretching of T4 DNA.

\section{Introduction}

Engineering DNA stretching processes and understanding the dynamics of DNA stretching are key steps in the development of the next generation of biological microfluidic (BioMEMs) devices. Many current applications like criminal DNA "fingerprinting" and medical diagnostics rely on a very low resolution view of a DNA's gene map. ${ }^{1}$ However, genetic information is inherently difficult to extract directly from DNA molecules because, in the size range of $\mathrm{O}[10 \mathrm{kbp}]$ and above, they adopt "random coil" configurations. Thus, if certain gene locations can be tagged with a fluorescent probe, a detection device cannot accurately determine the linear distance between the respective genes along the DNA backbone. The coil must first be unraveled and stretched. ${ }^{2,3}$ Access to a long strand of stretched DNA has recently led to powerful applications for gene mapping. ${ }^{2-8}$

Researchers have stretched tethered double-stranded DNA (dsDNA) with optical tweezers, ${ }^{9}$ uniform flows, ${ }^{10}$ and uniform electric fields. ${ }^{11}$ However, tethering a DNA molecule is an involved and not easily integrated step in a process design. Consequently, research groups are trying to completely stretch free DNA in a microchannel. Some current techniques are flowing DNA through a channel contraction, ${ }^{2,3,12}$ forcing DNA into a nanochannel, ${ }^{8,13}$ hydrodynamic focusing of multiple streams, ${ }^{14}$ dynamic combing onto a surface, ${ }^{4-7}$ or applying an AC field to DNA in a gel. ${ }^{15,16}$ From an engineering perspective, it is most attractive to stretch DNA in a continuous process. One such promising application is direct linear analysis (DLA), ${ }^{2,3,12}$ which entails binding probes to known sequences along the strand of DNA, stretching the

\footnotetext{
${ }^{a}$ Department of Chemical Engineering, Massachusetts Institute of Technology, MIT Room 66-456, 77 Massachusetts Ave., Cambridge, MA 02139, USA. E-mail: pdoyle@mit.edu; Fax: (617) 258-5042, Tel: (617) 253-4534

${ }^{b}$ Department of Chemical Engineering, Northeastern University, Boston, MA 02115, USA
}

molecule from a coiled to a linear conformation, and then directly measuring the distance between these probes continuously in-line. In current DLA designs, DNA is stretched by flow gradients as it moves through a channel contraction. However, more generally, a DNA macromolecule driven by any external field (e.g. a flow or an electric field) stretches in the presence of strong field gradients. These gradients may be induced by obstacles, channel contractions, or even surface modifications. Continuous stretching of DNA in field gradients has been studied in homogeneous hydrodynamic flows (like extensional flow, ${ }^{17,18}$ shear, ${ }^{19}$ or mixed flow ${ }^{20,21}$ ), and electric fields. ${ }^{22-25} \uparrow$ The main problems to overcome in order to stretch DNA continuously are shear $^{3}$ and molecular individualism. ${ }^{17,26}$

Shear, which arises in fluid flows near any surface due to the no slip condition, induces tumbling motion as opposed to strong extensional deformation. ${ }^{19}$ In a pressure-driven flow microdevice with a high surface area to volume ratio, shear rates near walls can often be much higher than strain rates. As these DNA stretching devices move to the nanoscale it will be important to eliminate this surface induced shear, though as explained below, some "pre-shear" may actually be useful to aid stretching. ${ }^{27}$ We showed previously ${ }^{22-24}$ that electrophoretic deformation is purely elongational without any rotational components. Thus by using electric field gradients to stretch DNA, very high electrophoretic strain rates can be achieved in microdevices without any shear. Electric fields are also easily implemented by placing electrodes in reservoirs at the ends of the microchannel. Furthermore, they have the additional advantages over pressure-driven flows at nanoscopic dimensions of avoiding high pressure-related channel failure and viscoelastic instabilities like corner vortex formation.

$\dagger$ We note that the value of the DNA mobility in the $30 \mathrm{cP}$ buffer presented in ref. $25\left(-1.16 \frac{\mu \mathrm{m}}{\mathrm{s}} / \frac{\mathrm{V}}{\mathrm{cm}}\right)$ is an order of magnitude too high. Thus it is doubtful that the observed stretching was electrophoretically induced. 
The other primary problem for continuous stretching of DNA is molecular individualism, ${ }^{17,26}$ a term used to describe the high sensitivity of DNA stretching dynamics in an extensional flow to its initial configuration. An ideal DLA device would be able to fully stretch every DNA molecule uniformly, but the highly configuration-sensitive dynamics makes this impossible when a molecule experiences a finite strain. Often a strain of at least 10 is needed for the majority of DNA in an ensemble to reach steadystate extension in extensional flows, ${ }^{28}$ and as we explain below, it is difficult to construct devices to yield such large strain. However, it is possible to control molecular individualism effects on the stretching dynamics. Larson ${ }^{27}$ used Brownian dynamics simulations to show that shifting the initial configurational distribution before applying an extensional flow can increase average DNA stretching for finite strain. Specifically, he studied DNA stretching in a two-step process: first imposing shear flow and then immediately thereafter an extensional flow. At strong extensional flow conditions, he found that some pre-sheared ensembles achieved a near uniform fractional extension after a strain of 4, whereas molecules simultaneously sheared and extended (with shear rate $\leqslant$ strain rate) showed the characteristic broad extension distribution and lower mean extension resulting from molecular individualistic effects. We seek to employ this general methodology of preconditioning the initial configurational distribution in order to reliably and uniformly stretch DNA.

In this work, we use single molecule fluorescence microscopy to study electrically-driven T4 DNA (169 kbp) extending in a hyperbolic contraction, migrating through a porous gel, and doing both in a hybrid gel/contraction channel where we pattern a porous UV crosslinked gel at the inlet of the contraction. The hyperbolic geometry is chosen because, barring entrance effects, the strain rate and primary axis of extension are constant throughout the contraction. The patterned gel causes the molecules to reptate and adopt extended configurations before entering the ontraction. We will show that DNA can fully and uniformly stretch when the gel biases the DNA configurations before entering the extensional electrical field.

\section{Background}

We refer the reader to our previous $\operatorname{paper}^{24}$ for a detailed background of DNA deformation in electric field gradients, which we briefly summarize here. Our analysis considers large double-stranded DNA molecules, typically of size $50-1000 \mathrm{kbp}$ ( $\sim 0.01-1 \%$ of a typical human chromosome), and free of all in vivo proteins. The DNA molecule can be modeled as a polymer and is primarily characterized by its persistence length $l_{\text {p }}$, contour length $L$, diffusivity $D$, and longest relaxation time $\tau$. The large DNA molecules considered here have $L \gg l_{\mathrm{p}}$ so that they adopt coiled configurations (with radius of gyration $R_{\mathrm{g}}$ ) at equilibrium in aqueous solvents. Backbone phosphate groups render DNA a uniformly negatively charged polymer at moderate $\mathrm{pH}$. In the presence of a uniform electric field $\boldsymbol{E}$, DNA coils move through solution at a size-independent velocity $\mu \boldsymbol{E}$ where $\mu$ is the electrophoretic mobility. ${ }^{29}$ In an electric field gradient, charged polymers can deform from their native coil configurations. With a bead-spring model of a polyelectrolyte in a low Debye length $\left(\kappa^{-1}\right)$ solvent with $\kappa^{-1} \ll l_{\mathrm{p}}$, deformation in electrophoretic fields $\mu \boldsymbol{E}$ and hydrodynamic flows $\boldsymbol{u}^{\infty}$ is equivalent (considering linear electrophoresis). ${ }^{30}$ Consequently, at low fields, we can use the same kinematic approach to study electrophoretic deformation as has been used in the past to study hydrodynamic deformation. The governing dimensionless group is the Deborah number $D e=\dot{\varepsilon}^{\mathrm{EL}} \tau$ where $\dot{\varepsilon}^{\mathrm{EL}}$ is the electrophoretic strain rate (positive eigenvalue of $\mu \nabla \boldsymbol{E}$ ) and $\tau$ is the longest relaxation time of the polymer. At a critical value of $D e=1 / 2$, polymer deformation behavior transitions to strong extension in a homogeneous field. Ideal or "affine" deformation occurs when two material charges with mobility $\mu$ initially separated by a small distance $\delta$ move apart exponentially in this field, so that their distance apart at a later time is $\delta \exp \left(\varepsilon^{\mathrm{EL}}\right)$, where ${ }_{\varepsilon}^{\mathrm{EL}}=\int \dot{\varepsilon}^{\mathrm{EL}} \mathrm{d} t$ is the accumulated electrophoretic strain. Polymers often do not stretch ideally due to their elasticity and conformation folds and kinks, so it is customary to compare the actual extension-strain of a DNA ensemble in relation to the affine scaling.

The above analysis applies nicely at low external fields, however because electric and hydrodynamic forces and flows are intimately coupled, complex nonlinear electrophoretic effects may arise at high field strengths $\left(\mathrm{O}\left[\mathrm{kV} \mathrm{cm}^{-1}\right]\right)$. These nonlinear electrophoretic effects arise when the double layer of the moving object becomes polarized. Polarization induces secondary fields and flows that affect the mobility ${ }^{31-34}$ and also imposes a dielectrophoretic body force. Previous dielectrophoresis research has shown that DNA molecules do indeed polarize positively in AC fields, ${ }^{35-37}$ and can even exhibit polarization-induced aggregation both in high AC or DC fields. ${ }^{38-40}$ In our experiments, we use field strengths and molecule sizes where the dielectrophoretic force and other induced nonlinear effects are negligible. ${ }^{41}$

\section{Device characterization}

We investigate the stretching of DNA molecules in a hyperbolic contraction microchannel as shown in Fig. 1. In this channel (Fig. 1a), DNA are driven by an electric field to the hyperbolic contraction from the right down a wide inlet channel of width $w_{1}$ and length $l_{1}$. The sidewalls then contract according to a hyperbolic equation of the form: $y=C /\left(x+2 C / w_{1}\right)$ where $x$ and $y$ are a set of coordinates with the origin along the symmetry line of the channel at the point where the hyperbolic begins and the inlet ends (Fig. 1b). The length of the contraction is $l_{\mathrm{c}}$. After moving through the contraction, the DNA then migrate down a narrow channel of width $w_{2}$ and length $l_{2}$, and afterwards move though an abrupt expansion and travel to a waste reservoir down a channel of length $w_{3}$ and length $l_{3}$. The dimensions used in this study were: $w_{1}=200 \mu \mathrm{m}, w_{2}=3.8 \mu \mathrm{m}$, $w_{3}=200 \mu \mathrm{m}, l_{1} \sim 1.5 \mathrm{~mm}, l_{2}=1.52 \mathrm{~mm}, l_{3} \sim 1.5 \mathrm{~mm}$, and $l_{\mathrm{c}}=$ $80 \mu \mathrm{m}$. The hyperbolic constant $C\left(=w_{2} l_{\mathrm{c}} /\left(2-2 w_{2} / w_{1}\right)\right)$ was $155 \mu^{2}$. 

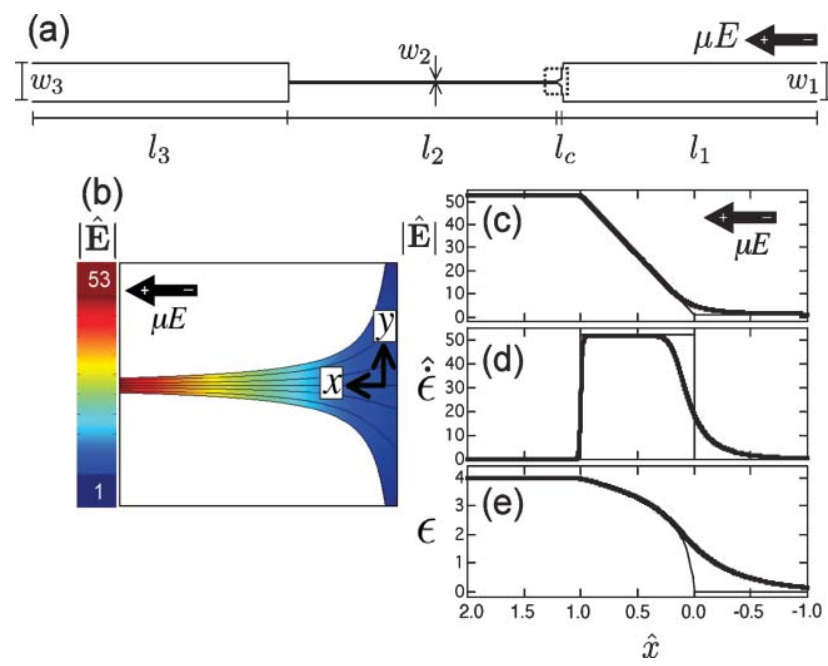

Fig. 1 (a) Diagram of the hyperbolic contraction geometry. The vector $\mu \boldsymbol{E}$ points in the direction of DNA motion (right to left). (b) Dimensionless electric field strength in the hyperbolic region from finite element calculation. (c) Dimensionless electric field strength along the $y=0$ trajectory. (d) Dimensionless strain rate along the $y=0$ trajectory. (e) Strain accumulated along the $y=0$ trajectory. The thin lines in (c)-(e) are for an ideal hyperbolic contraction without entrance or exit effects.

For the remaining analysis, we will non-dimensionalize the downstream position, electrophoretic velocity, and electrophoretic strain rate as:

$$
\begin{aligned}
\widehat{x} & =\frac{x}{l_{c}} \\
\widehat{\boldsymbol{E}} & =\frac{\boldsymbol{E}}{E_{1}} \\
\widehat{\dot{\varepsilon}} & =\dot{\varepsilon}^{\mathrm{EL}} \frac{l_{c}}{\mu E_{1}}
\end{aligned}
$$

where $E_{1}$ is the uniform electric field in the inlet region of length $l_{1}$ and we have dropped the EL superscript for nondimensional electrophoretic strain rate. We will similarly drop the EL superscript to report electrophoretic strain.

In Fig. 1b we show a finite element calculation of the 2-dimensional (2D) electric field strength $|\widehat{\boldsymbol{E}}|$ along with expected trajectories in the hyperbolic section of the channel. The molecules move right to left and accelerate through the hyperbolic to a maximum electrophoretic velocity in the narrow section of the channel. Fig. 1c shows the scaled electrophoretic velocity in the hyperbolic region of the channel from the finite element computation. This data is for the $y=0$ trajectory, however we confirmed that other trajectories exhibit a similar velocity trend. The electrophoretic velocity for an idealized hyperbolic channel without any end effects is indicated by the thin line. Note that for this device the velocity is $\sim 53$ times greater in the narrow section than at the inlet and there is an approximately constant velocity gradient in the hyperbolic region. There are however entrance and end effects that are more evident in Fig. 1d, which shows $\partial|\widehat{\boldsymbol{E}}| / \partial \widehat{x}(=\widehat{\hat{\varepsilon}})$. Again, the strain rate for an ideal channel without entrance or exit effects is indicated by a thin line. Because of the large change in channel dimensions, the entrance region extends a full contraction length before the contraction begins and persists into about $25 \%$ of the contraction length. At this point the strain rate is constant until about the last $5 \%$ of the contraction, at which point it abruptly falls to zero. Fig. 1e shows the electrophoretic strain integrated along the $y=0$ trajectory ( $s$ is the distance along the trajectory path):

$$
\begin{aligned}
\varepsilon & =\int_{\text {path }} \frac{\dot{\varepsilon}(s) \mathrm{d} s}{|\mu \boldsymbol{E}|(s)} \\
& =\int_{(-\infty, 0)}^{(\widehat{x}, 0)} \frac{\widehat{\dot{\varepsilon}}(\widehat{x}) \mathrm{d} \widehat{x}}{|\mu \widehat{\boldsymbol{E}}|}
\end{aligned}
$$

along with the strain of the ideal hyperbolic channel $\left(\ln \left[\left(\widehat{x}+C /\left(l_{\mathrm{c}} w_{1}\right)\right) /\left(C /\left(l_{\mathrm{c}} w_{1}\right)\right)\right]\right)$. Note that this geometry allows for a total strain of 4 , however a significant strain $(\varepsilon \sim 2)$ is achieved in the nonhomogeneous entrance region. A similar nonhomogeneous field was treated in ref. 24 and a general treatment of deformation in non-homogeneous fields can be found in ref. 42. The primary effect of the nonhomogeneous entrance region will be to reduce the effective strain experienced in the strong extensional region ( $D e>1 / 2)$ when $D e$ is low. Additionally, it will also slightly shift the DNA's configuration distribution at the hyperbolic inlet to slightly more extended states. ${ }^{24}$ However, we stress that this shift is far less than the shift required to sufficiently avoid molecular individualistic effects.

\section{Experimental}

We used soft lithography ${ }^{43}$ to construct $2 \mu \mathrm{m}$-high PDMS (polydimethylsiloxane) microchannels with geometry as described above. A 10:1 PDMS crosslinking agent (Sylgard 184, Dow) was degassed for $60 \mathrm{~min}$ at $15^{\prime \prime} \mathrm{Hg}$ vacuum pressure. The PDMS was then poured onto a Si master wafer with the negative of the microchannel geometry embedded in a layer of AZ 5214 image reversal photoresist (Clariant). The Si wafer was pretreated with a fluorinated silane monolayer (United Chemical Technologies) to prevent cured PDMS from sticking to the $\mathrm{Si}$ master. After pouring, the PDMS was allowed to degas for an additional hour at $15^{\prime \prime} \mathrm{Hg}$. The PDMS was then cured at $65^{\circ} \mathrm{C}$ for $24 \mathrm{~h}$. Reservoirs $(4 \mathrm{~mm} \times 4 \mathrm{~mm})$ were cut at each end of the cured PDMS microchannel with a scalpel. Fig. 2a shows an SEM image of a typical hyperbolic channel.

T4 DNA (169 kbp, $L=70 \mu \mathrm{m}$ stained, $\left.R_{\mathrm{g}} \sim 1.5 \mu \mathrm{m}\right)$ were stained with a fluorescent dye (TOTO-1, $4.7: 1$ bp : dye molecule) and diluted in one of the following buffers: $5 \times \mathrm{TBE}$, $4 \% \quad \beta$-mercaptoethanol, and $0.1 \%$ polyvinylpyrrolidone $(\mathrm{PVP}$, Polysciences, $\mathrm{MW}=10000)(\eta=1 \mathrm{cP}, \mathrm{pH}=8.3)$ or $5 \times \quad$ TBE, $4 \% \quad \beta$-mercaptoethanol, $0.1 \% \quad \mathrm{PVP}$ (Polysciences, MW $=10000)$, and $31 \%$ sucrose $(\eta=6 \mathrm{cP}$, $\mathrm{pH}=7.9)$. The $6 \mathrm{cP}$ buffer was only used for our $D e=$ 23 study to slow down dynamics to experimentally observable speeds. The PVP is added to the formulation to dynamically coat the microchannel walls to minimize electroosmotic flow. The measured electrophoretic mobility of the DNA was $\mu=-1.3 \pm 0.2 \frac{\mu \mathrm{m}}{\mathrm{s}} / \frac{\mathrm{V}}{\mathrm{cm}}$ in the $1 \mathrm{cP}$ buffer and $\mu=-0.17 \pm 0.02 \frac{\mu \mathrm{m}}{\mathrm{s}} / \frac{\mathrm{V}}{\mathrm{cm}}$ in the $6 \mathrm{cP}$ buffer. We 


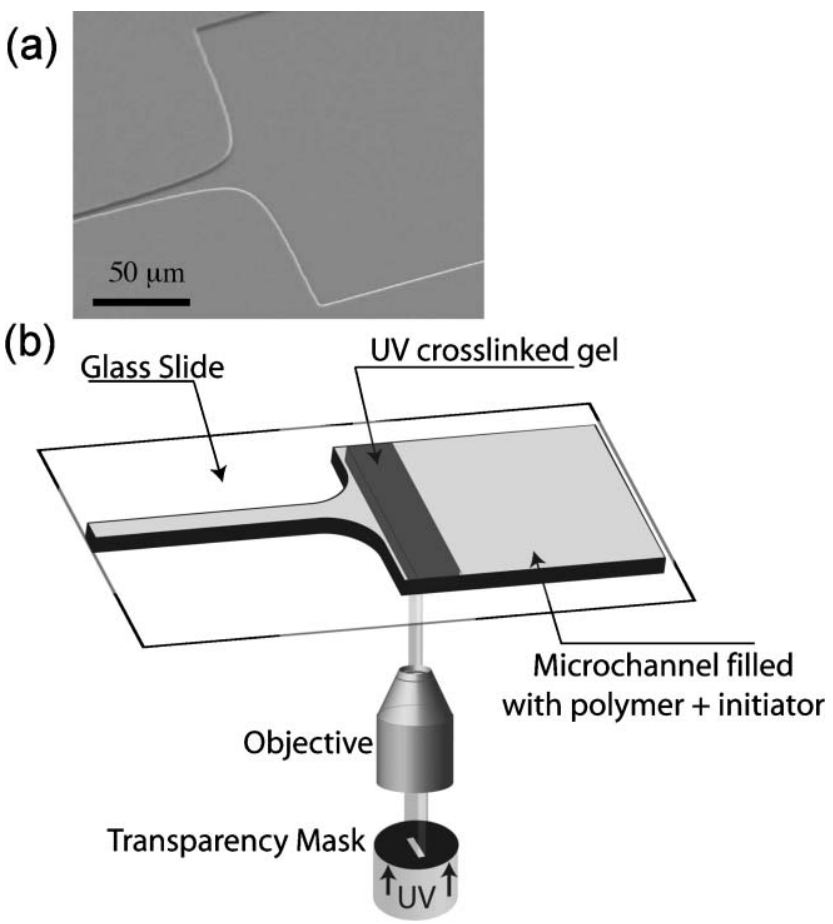

Fig. 2 (a) SEM image of a PDMS hyperbolic contraction. (b) Schematic of the selective UV crosslinking procedure used to fabricate gels at the entrance to the hyperbolic contraction (not to scale).

employ the convention of using a minus sign for DNA mobility so that $\mu \boldsymbol{E}$, which points in the direction of DNA motion, is in the opposite direction of $\boldsymbol{E}$. By applying an electric field and tracking very low-charged, surfactant-stabilized emulsion droplets $\left(R_{\text {bead }}=\right.$ $0.25 \mu \mathrm{m}$, Ademtech, Pessac, France) in our $1 \mathrm{cP}$ buffer in a control experiment, we calculate the background electroosmotic mobility to be $\mu_{\mathrm{EOF}}=0.15 \frac{\mu \mathrm{m}}{\mathrm{s}} / \frac{\mathrm{V}}{\mathrm{cm}}$. We measure the contour length by molecular combing ${ }^{44}$ and observations of stretched tethered configurations at $\mathrm{Pe}\left(=\mu E l_{\mathrm{p}} / D\right) \sim 100$. The measurement of $L=$ $70 \mu \mathrm{m}$ was later verified by our consistent hyperbolic stretching experiments. By measuring the dynamic mean square extension of 15 stretched T4 DNA molecules as they relaxed in a $2 \mu \mathrm{m}$-thin PDMS microchannel as in ref. 24, we determined the longest relaxation time for T4 DNA in these channels to be $\tau=1.7 \mathrm{~s}$ in the $1 \mathrm{cP}$ buffer. The DNA were fairly monodisperse, though some break during storage and handling. Approximately 2/3 of the DNA coils are intact T4 molecules in these experiments. Broken molecules are generally easy to spot as they tend to be approximately half the size of an intact $\mathrm{T} 4$ molecule. Nevertheless, a possible source for error in these studies is the quality of the DNA ensemble.

\section{Experiments without gels}

A typical experiment without a gel consisted of first soaking the PDMS channel for $12 \mathrm{~h}$ at $45^{\circ} \mathrm{C}$ in $0.5 \times \mathrm{TBE}$ to eliminate permeation driven flow. ${ }^{45}$ Next, we gently rinsed and dried the microchannel and applied it to a clean glass slide (soaked in $1 \mathrm{M} \mathrm{NaOH}$ for $15 \mathrm{~min}$ and rinsed in ultrapure water (MilliQ, Millipore)). We then immediately filled the channel with DNA solution and applied an electric field across the reservoirs through platinum electrodes. After 15 minutes of equilibration, we observed single DNA molecule dynamics using an inverted fluorescence microscope (Axiovert 200, Zeiss) with a $63 \times 1.4$ NA objective and $100 \mathrm{~W}$ mercury lamp light source operating at 50\% intensity (HBO 103, Zeiss). Images were captured at 30 frames per second with an EB-CCD camera (C7190-20, Hamamatsu) and NIH Image software. Digitized images had 8-bit pixel intensity values which ranged from $0-255$. The primary experimental observable is the extension of the DNA $x_{\mathrm{ex}}$. We define $x_{\mathrm{ex}}$ as the maximum linear dimension of the DNA's fluorescence cloud. ${ }^{24}$ For a stretched molecule, $x_{\mathrm{ex}}$ is simply the distance between the front $\left(x_{\mathrm{f}}, y_{\mathrm{f}}\right)$ and back coordinates $\left(x_{\mathrm{b}}, y_{\mathrm{b}}\right)$. We manually adjust for the effect of a curved contour length in the hyperbolic for any molecules that stretch over $40 \mu \mathrm{m}$ long with a back coordinate $|y|>20 \mu \mathrm{m}$. An equilibrium T4 coil in the inlet section of a $2 \mu \mathrm{m}$-high channel had a mean maximum linear dimension $\left\langle x_{\mathrm{ex}}\right\rangle=$ $4.6 \pm 0.8 \mu \mathrm{m}\left(<x_{\mathrm{ex}} / L>=0.07\right)$.

\section{Experiments with gels}

In some experiments, we selectively crosslink a porous gel directly before the hyperbolic contraction. To do this we first soak the PDMS channel in an initiator solution of $10 \%$ 1-hydroxycyclohexyl phenyl ketone (Aldrich, 405612) in 70\% ethanol and ultrapure water for $30 \mathrm{~min}$. We then rinse the channel in ultrapure water, going through a cycle three times of rinsing in a water bath and then sonicating for 20 s. After rinsing, we let the PDMS soak in an ultrapure water bath for 30 min and then carefully dry the PDMS and a glass slide (as cleaned without gel). We then treat both the PDMS and glass in a RF plasma cleaner (Harrick, PDC-32G) for $5 \mathrm{~s}$ at $60 \mathrm{~W}$ and bond the two together. We speculate that this bonding step is necessary when patterning gels in these channels because the gel may swell in aqueous buffers and cause liftoff. The remaining gel patterning steps ${ }^{46}$ are shown schematically in Fig. 2b. Once bonded, we fill the channel with $5 \mu \mathrm{L}$ of a solution of $2.5 \%$ poly(ethylene glycol) 1000 dimethacrylate ("PEG-DM 1000", Polysciences, 15178), 2\% poly(ethylene glycol) 400 diacrylate ("PEG-DA 400", Polysciences, 01871), 1\% 2-hydroxy-2-methyl-propiophenone ("Darocur 1173", Aldrich, 405655) in 50\% ethanol and 50\% $5 \times$ TBE. The PEG-DM 1000 and PEG-DA 400 monomers crosslink when in the presence of the Darocur 1173 initiator and UV light. After filling, we expose the intended gel region with $\sim 365 \mathrm{~nm} \mathrm{UV}$ light obtained with a UV excitation filter set (11000 v2 : UV, Chroma, Rockingham, VT) through a transparency mask (placed in the microscope's field stop aperture, CAD/Art Services Inc., Bandon, OR) and a $40 \times 0.75$ numerical aperature objective. The mask has an $8 \mathrm{~mm}$-long by $2 \mathrm{~mm}$ wide transparent region which creates a gel approximately $150 \mu \mathrm{m}$-wide after focussing through the objective. Exposure is performed in two steps: first a $3 \mathrm{~s}$ exposure, followed by a $10 \mathrm{~s}$ cooling time, and then a $2 \mathrm{~s}$ exposure. This two-step process is required to prevent the transparency mask from melting. Following exposure, we then remove the monomer solution from the loading reservoir and then fill both reservoirs with $0.5 \times$ TBE. We then overfill the outlet reservoir and apply a 
potential of $50 \mathrm{~V}$ across the reservoirs to force a combined pressure-driven and electroosmotic rinse flow from the outlet to the inlet of the microchannel for $30 \mathrm{~min}$. Following the rinse, we empty the reservoirs and then fill both reservoirs with the DNA solution. We allow for a 15 minute equilibration period with an applied voltage of $130 \mathrm{~V}$ to load DNA and buffer into the microchannel and to allow for quenching of the electroosmotic flow. The DNA observation and analysis then proceeded as in the experiments without gels.

\section{Field characterization}

We performed bead electrophoresis experiments to characterize the electric field in the hyperbolic contraction. A $0.01 \%$ solution of carboxylated polystyrene fluorescent beads (negatively charged, $R_{\text {bead }}=0.16 \mu \mathrm{m}$, Polysciences) was prepared in the $1 \mathrm{cP}$ buffer along with an additional $0.5 \%$ tergitol (NP-10, J. T. Baker) to prevent beads from sticking to the channel walls. The bead solution was loaded into a hyperbolic channel and an electric field was applied to drive the beads into the contraction. The maximum field strength at the contraction exit was $E_{2}=425 \mathrm{~V} \mathrm{~cm}^{-1}$, which is the near the highest field strength achieved in the DNA deformation study. Nonlinear electrophoretic effects discussed in ref. 31-34 are negligible at these conditions. We tracked the center of mass position of 25 beads. Their trajectories, which are predictably hyperbolic in shape, are shown in Fig. 3a. Fig. 3b shows a semi-log plot of the $x$-position of each bead versus time. The thick line is the affine scaling $\widehat{x} \sim \exp \left(\dot{\varepsilon}^{\mathrm{EL}} t\right)$ in the homogeneous part of the extensional field. For each bead trace we fit for an experimentally observable strain rate $\dot{\varepsilon}_{\text {obs }}^{\mathrm{EL}}$ with $\widehat{x}=\widehat{x}(0) \exp \left(\dot{\varepsilon}_{\text {obs }}^{\mathrm{EL}} t\right)$ from $\widehat{x}(0)=0.2$ up until $\widehat{x}(t)=0.9$. The ensemble average strain rate was determined to be $\left\langle\dot{\varepsilon}_{\text {obs }}^{\mathrm{EL}}\right\rangle=4.4 \pm 0.3 \mathrm{~s}^{-1}$. We also independently
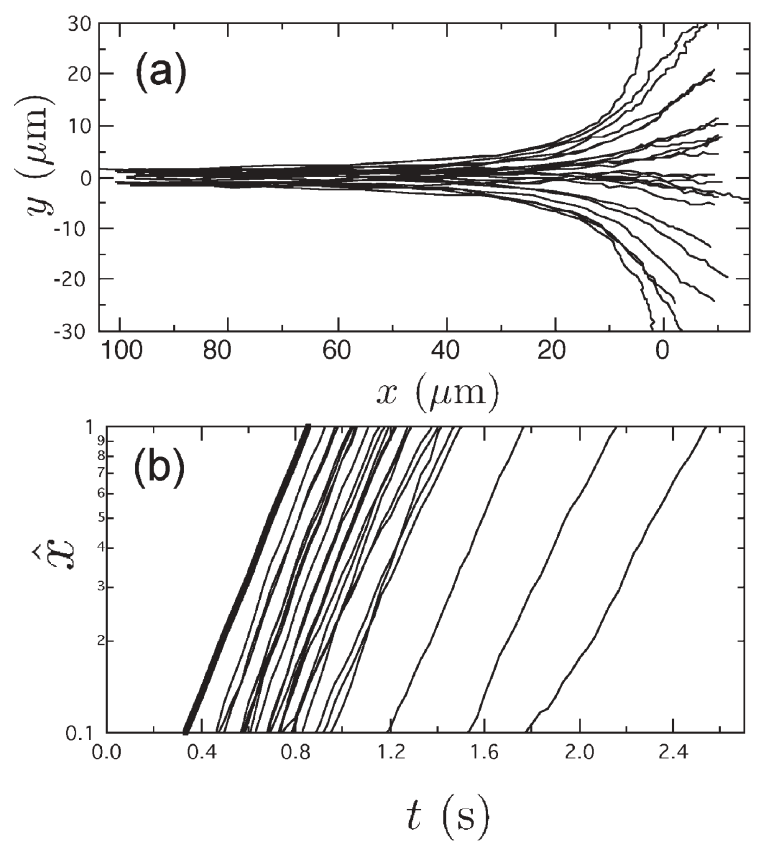

Fig. 3 (a) Trajectories of carboxylated bead electrophoresis for field characterization ( $\left.E_{2}=425 \mathrm{~V} \mathrm{~cm}^{-1}, R_{\text {bead }}=0.16 \mu \mathrm{m}, 1 \mathrm{cP}\right)$. (b) Semilog $\widehat{x}(\mathrm{t})$ traces for the above bead electrophoresis data. The thick line is the affine scaling using $\dot{\varepsilon}^{\mathrm{EL}}=4.4 \mathrm{~s}^{-1}$. measured the velocity of beads in the narrow section $\left(l_{2}\right)$ of the hyperbolic and then divided by the contraction length $l_{\mathrm{c}}$ to obtain $\left\langle\dot{\varepsilon}_{\text {obs }}^{\mathrm{EL}}\right\rangle=4.4 \pm 0.3 \mathrm{~s}^{-1}$. Hence each bead accelerates affinely, as expected for this well-behaved field. In DNA experiments, we determine the strain rate using the latter method, i.e. by measuring the velocity of DNA molecules in the narrow section $\left(l_{2}\right)$ of the device and computing $\dot{\varepsilon}^{\mathrm{EL}} \approx \mu E_{2} / l_{\mathrm{c}}$. Now that we have characterized the channel, we study how DNA coils deform in it.

\section{Results}

\section{Hyperbolic contraction}

Fig. $4 \mathrm{a}-\mathrm{d}$ shows the fractional extension $x_{\mathrm{ex}} / L$ of T4 DNA driven through the contraction by electric fields at $D e=2,7$, 14, and 23. The fractional extension data is plotted against both the $x$-coordinate of the front of the DNA molecule $\widehat{x}_{\mathrm{f}}=x_{\mathrm{f}} / l_{\mathrm{c}}$ (lower axis) and the electrophoretic strain (top axis) calculated from Fig. 1e. The strain experienced by a DNA molecule is difficult to rigorously define since a stretched T4 molecule can span a large fraction of the contraction and even sample different strain rates in the entrance region. Recall that electrophoretic strain is a measure of how ideal charged objects move exponentially apart in a field gradient. Because we are
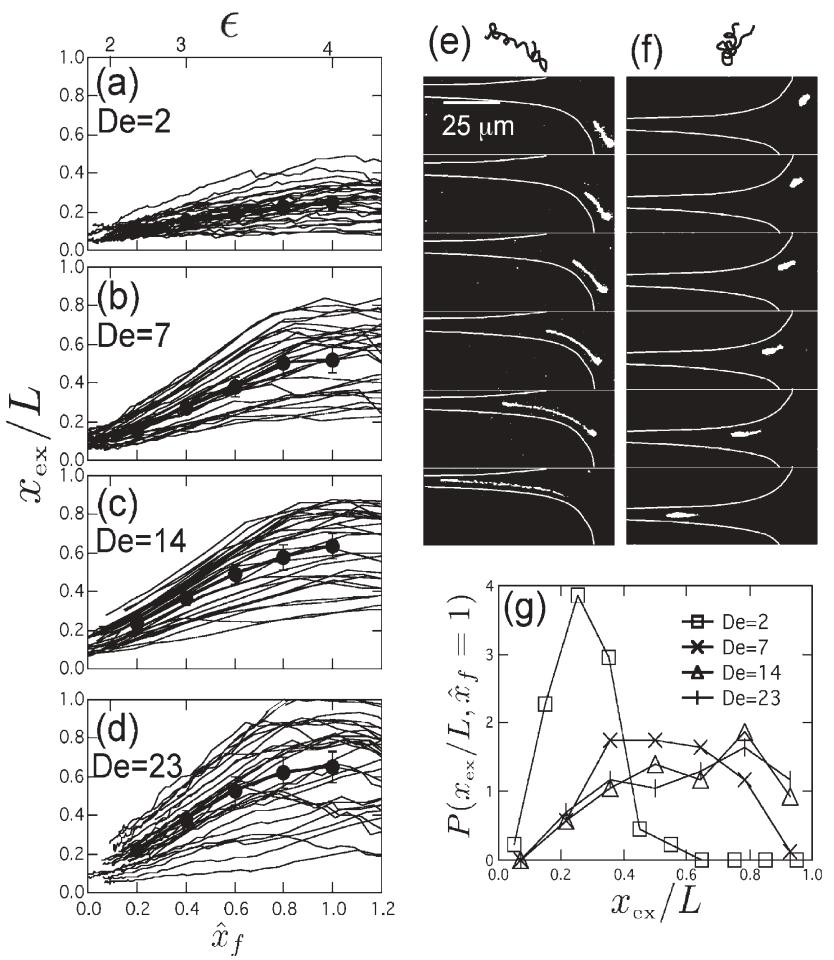

Fig. 4 Fractional extension of T4 DNA in the hyperbolic contraction (no gel) at (a) $D e=2$ (b) $D e=7$ (c) $D e=14$ and (d) $D e=23$ (ensemble sizes of $n=30$ shown and ensemble averages are given by solid circles). (e)-(f) Sample images (0.17 s timesteps between frames) of T4 DNA stretching in the hyperbolic contraction at $D e=7$. The hyperbolic sidewalls are drawn in for reference. (e) A "dumbbell" molecule in an initially open configuration. (f) A "folded" molecule in an initially closed configuration. (g) Probability distribution $(n=60)$ of T4 DNA fractional extension as its front leaves the hyperbolic contraction at each $D e$ studied. 
studying how the front of the DNA moves away from its back, we have chosen to plot the strain at the front coordinate of the molecule.

Given electrohydrodynamic equivalence, ${ }^{30}$ one might first expect the data to closely match results of ref. 17, 18 and 24 . However the field is not strictly homogeneous. Recall that $\widehat{\dot{\varepsilon}}$ ramps to its maximum value from $-1<\widehat{x}<0.25$ and also note that the extended lengths of these T4 molecules are on the order of the length of the contraction. We find that the $D e=2$ fractional extension is noticeably lower than expected, as previous experiments $^{17,18,24}$ and simulations ${ }^{28}$ show $\left\langle x_{\mathrm{ex}} / L\right\rangle \sim 0.5$ and some steady-state configurations with $x_{\mathrm{ex}} / L \sim 0.75$ after a strain of 4 . We attribute this difference primarily to the inhomogeneous entrance region where $\widehat{\dot{\varepsilon}}$ slowly ramps from 1 to 53 . At $D e=2$, the strong stretching criterion $(D e>1 / 2)$ is not achieved until $\widehat{x} \sim 0$. Thus, the accumulated 1.5 units of strain occurring before $\widehat{x} \sim 0$ cannot induce strong deformation, which decreases the strain experienced in the strong stretching regime to 2.5. Our ensemble average $\left(\left\langle x_{\mathrm{ex}} / L\right\rangle=0.26\right)$ corresponds closely to homogeneous flow studies ${ }^{18}$ after $\varepsilon=2.5$. We conclude that these molecules do not experience enough strain in the strong stretching regime for any to achieve steady-state extensions.

Conversely, at higher $D e$, the strong stretching condition is met well before the contraction and the molecules experience $\varepsilon \sim 4$. This is ample strain for some molecules in the ensemble to reach steady state extension at the outlet and the extension data more closely follows previously published results in flows ${ }^{18}$ (i.e. $\left\langle x_{\mathrm{ex}} / L>=0.6\right.$ at $D e=14$ after $\varepsilon=4$ ). Note that we have achieved these extension results in a $2 \mu \mathrm{m}$-thin channel. This close agreement also implies that the entrance region and DNA finite size effects are not significantly altering the ensemble stretching dynamics. Consequently we see a variety of extension behavior, with some molecules extending much faster than others. We demonstrate this individualistic behavior by examining two particular configurations in Fig. 4e and $f$, which show individual DNA molecules stretching at $D e=7$. Fig. 4 e shows a T4 DNA molecule that enters the contraction in an open dumbbell-like configura$\operatorname{tion}^{17,18}$ and then significantly stretches. However Fig. 4f shows a T4 DNA molecule that enters in a compact coiled configuration $^{17,18}$ and does not stretch much in the contraction. This sensitive dependence of stretching on the initial configuration makes stretching DNA uniformly at a moderate strain impossible.

Fig. $4 \mathrm{~g}$ shows the fractional extension probability distributions for T4 when the front of its fluorescence cloud exits the hyperbolic $\left(\widehat{x}_{f}=1\right)$ at various $D e$. Table 1 reports each ensemble's mean and standard deviation. It is clear that as $D e$

Table 1 Summary of ensemble average fractional extension and standard deviation at the contraction exit

\begin{tabular}{ll}
\hline$D e$ & $\left\langle x_{\mathrm{ex}} / L\right\rangle$ at $\widehat{x}_{\mathrm{f}}=1$ \\
\hline 2 & $0.26 \pm 0.10$ \\
7 & $0.54 \pm 0.17$ \\
14 & $0.61 \pm 0.21$ \\
23 & $0.60 \pm 0.24$ \\
2, gel & $0.71 \pm 0.13$ \\
14, gel & $0.95 \pm 0.08$ \\
\hline
\end{tabular}

increases, the distributions shift to higher extensions. However, using kink dynamics arguments, ${ }^{47}$ one might expect this shift to saturate around $D e=40$. Judging by the similarity in the $D e=14$ and $D e=23$ data, it is clear that our study comes close to this saturation point. Hence, in a finite-strain hyperbolic contraction, though some DNA may stretch near full contour length, other DNA will only weakly stretch. This leads to a broad extension probability distribution even at high $D e$.

\section{UV crosslinked gel and uniform electric fields}

In order to more uniformly stretch DNA, we seek to preconfigure the configuration ensemble to more dumbbelllike open configurations before they enter the contraction as in Fig. 4e. The method we choose is to force the DNA into a crosslinked porous gel which we are able to pattern anywhere inside a microchannel. Before we do that, we first investigate the DNA deformation dynamics in a $150 \mu$ m-wide gel in a straight-walled channel with a uniform electric field ( $E=10 \mathrm{~V} \mathrm{~cm}^{-1}$, equivalent to the inlet field $E_{1}$ in $D e=$ 14 experiments).

The gel has pore sizes of $\mathrm{O}\left[l_{\mathrm{p}}\right]$ which will force each molecule to adopt more extended configurations in order to reptate through. Fig. 5a shows a time series of images of a T4 DNA moving through the gel. We immediately see that on average, the DNA adopt extended so-called "I-shape" configurations and traverse the pores in the same manner as observed in concentrated agarose or polyacrylamide solutions. ${ }^{15,48,49}$ This is best seen by observing the third, fourth, and fifth images of Fig. 5a.

Though the DNA do adopt extended configurations while reptating in the gel, they stretch even more as they leave. In a uniform field, DNA stretching is induced by imposing a tethering force. The gel provides the tethering effect, much like the large "drag tags" of end-labeled free solution electrophoresis (ELFSE) $)^{50,51}$ or by driving DNA into a porous matrix. ${ }^{52}$ The stretching at the exit occurs because the average mobility in the gel (at $E=10 \mathrm{~V} \mathrm{~cm}^{-1}$ ) is less than the mobility in free solution. However, the instantaneous mobility difference may vary significantly depending on the DNA's configuration. In an extreme example, some molecules act like they are tethered at the back due to formation of hairpin hooks around pieces of the gel. Fig. 5b shows an example of a T4 DNA exiting the gel in the uniform field, where the back end of the molecule becomes hooked on a piece of the gel and forms a hairpin. Many other exiting configurations are possible, e.g. dumbbells, half-dumbbells, or leading folds. ${ }^{17,18}$ Fig. 5c shows the fractional extension probability distribution of an ensemble of 100 T4 DNA molecules both inside the gel and as its back end exits the gel at $E=10 \mathrm{~V} \mathrm{~cm}^{-1}$. Note that the configuration distribution has shifted to moderately extended states $\left(<x_{\mathrm{ex}} / L\right\rangle$ $=0.21 \pm 0.11)$ inside the gel (versus $\left\langle x_{\mathrm{ex}} / L\right\rangle=0.07 \pm 0.01$ in a channel without the gel). Furthermore, the DNA stretch even more as they leave the gel $\left(\left\langle x_{\mathrm{ex}} / L\right\rangle=0.44 \pm 0.13\right)$. These DNA do not completely stretch, though it may be possible to achieve high stretching by increasing the field. However, the gel exit stretching distribution is still qualitatively broad, indicating that the details of an individual exiting DNA's configuration are still greatly influencing the stretching 


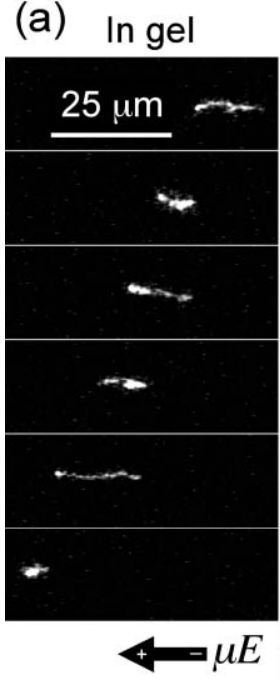

(b)

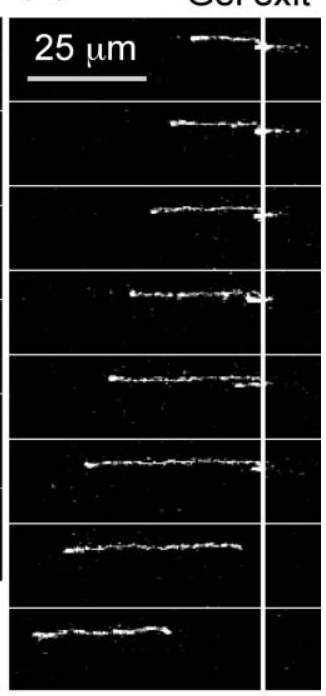

(c)

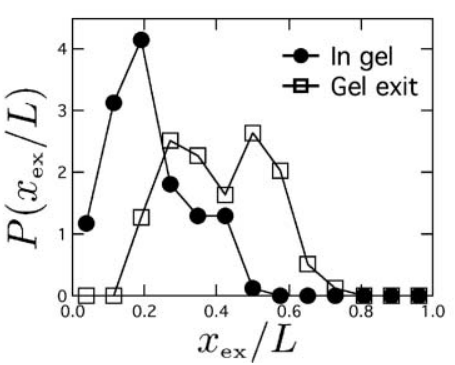

Fig. 5 Images and data for a T4 DNA in the UV crosslinked gel in a straightwalled microchannel (uniform electric field) and exiting the UV crosslinked gel in the same channel (different molecule). The channel has dimensions of height $=2 \mu \mathrm{m}$, width $=200 \mu \mathrm{m}$, length $=4.9 \mathrm{~mm}$ and $E=10 \mathrm{~V} \mathrm{~cm}^{-1}$. (a) Images (spaced $6.7 \mathrm{~s}$ apart) of DNA moving through the gel. (b) Images (spaced $0.6 \mathrm{~s}$ apart) of DNA exiting the gel. The vertical white line marks the end of the gel. (c) Probability distributions $(n=100)$ of T4 DNA fractional extension within and exiting this $\mathrm{UV}$ crosslinked gel at $E=10 \mathrm{~V} \mathrm{~cm}^{-1}$.

dynamics. A more attractive method to complete and uniform stretching is to introduce these prestretched DNA to an extensional field.

\section{Hybrid gel/contraction}

In the following experiments we selectively UV crosslink a $150 \mu \mathrm{m}$-wide gel before the entrance to the hyperbolic contraction as in Fig. 2b (gel ends at $\widehat{x}=-0.13$ ). Fig. 6a-d show a comparison of T4 DNA fractional extension without the gel and with the gel at $D e=2$ and 14 . Note that for both $D e$, the gel causes a large majority of the ensemble to strongly stretch before the hyperbolic exit. This is seen more clearly in Fig. 6e which shows the fractional extensional probability distributions at $\widehat{x}_{\mathrm{f}}=1$. The very broad extension distributions without the gel have shifted to narrow spikes with the gel $\left(\left\langle x_{\mathrm{ex}} / L\right\rangle=0.71 \pm 0.13\right.$ at $D e=2$ and $\left\langle x_{\mathrm{ex}} / L\right\rangle=0.95 \pm 0.08$ at $\left.D e=14\right)$. The stretching results at $D e=14$ had particularly excellent uniformity, as $80 \%$ of the ensemble stretched over $x_{\mathrm{ex}} / L=0.96$ and only $7 \%$ of the ensemble stretched less than $x_{\mathrm{ex}} / L=0.80$. We confirmed that these outliers corresponded to molecules that had a leading fold in their configuration as they exited the gel. Fig. 6f shows a series of
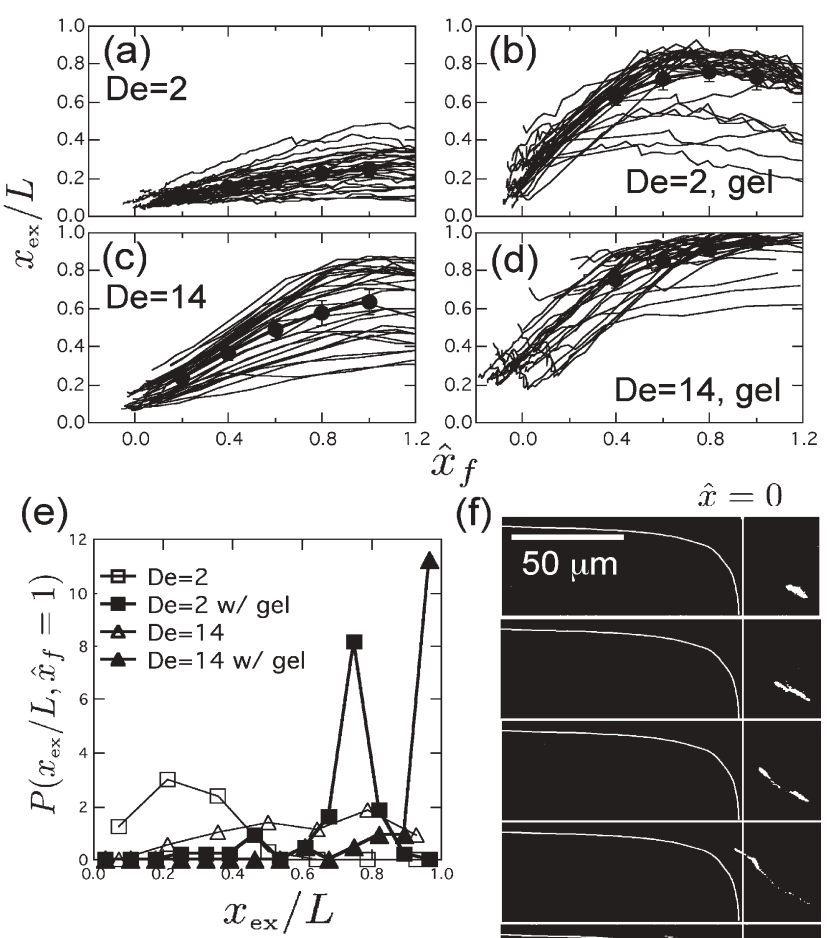

(f)

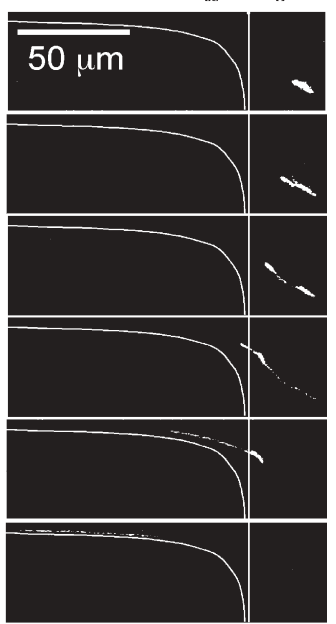

Fig. 6 Fractional extension of T4 DNA extending at (a) $D e=2$ in the hyperbolic contraction, (b) $D e=2$ in the contraction/gel hybrid channel, (c) $D e=14$ in the hyperbolic contraction, (d) $D e=14$ in the contraction/gel hybrid channel. Ensemble sizes of $n=30$ are shown and ensemble averages are given by solid circles. (e) Comparision of the fractional extension probability distributions $(n=60)$ at $\widehat{x}_{\mathrm{f}}=1$ for cases with and without the UV gel in front of the contraction. (f) Sample images of T4 DNA exiting the gel (its left edge is at $\widehat{x}=-0.13$ ) and entering the hyperbolic contraction at $D e=14$. A white line is drawn at $\hat{x}=0$ for reference. All frames are spaced by $0.67 \mathrm{~s}$ except the last two which are separated by $0.23 \mathrm{~s}$.

snapshots of a T4 molecule stretching as it exits the gel and enters the hyperbolic contraction at $D e=14$.

\section{Discussion}

With a hybrid gel-microcontraction device we are able to continuously obtain highly uniform DNA stretching using electric fields. This device overcomes the primary obstacles to continuous stretching of DNA: shear and molecular individualism. Shear is eliminated by using electric field gradients to deform the DNA. Consequently we are able to achieve comparable stretching results in a thin $2 \mu \mathrm{m}$-channel (without the gel) as previously shown in extensional flows far from any walls. However molecular individualism is still apparent because the strain is only $\varepsilon=4$. Recall that $\varepsilon>10$ is generally required for an entire ensemble of polymers to stretch in a uniform extensional flow or field. However, because $\varepsilon \sim \ln \left(w_{1} / w_{2}\right)$, it is extremely difficult to construct devices with $\varepsilon>10$. For example, decreasing the width of $w_{2}$ by a factor of 10 to $380 \mathrm{~nm}$ 
(a)

(b)
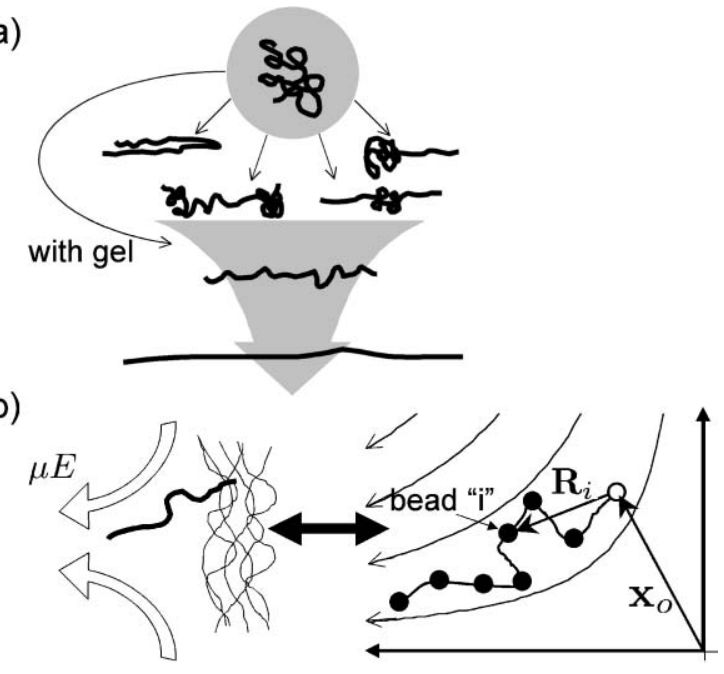

Fig. 7 (a) Schematic representation of the configuration manifold of a polymer coil transitioning to high extension (with and without preconditioning). (b) Sketch of a DNA molecule exiting a gel into an extensional field and the "pseudo-tethered" chain model.

would only increase the strain to $\varepsilon \sim 6$. Consequently, continuous and uniform stretching is not practically possible without a way to circumvent the effects of conformational molecular individualism. The hybrid gel/contraction is able to uniformly stretch DNA at moderate strain because (1) the gel shifts the entering configuration to mostly untangled stretched states (i.e. controlling molecular individualism) and (2) it acts as a pseudo-tether for the back of the molecule. Fig. $7 \mathrm{a}$ and $\mathrm{b}$ shows a schematic of each of these effects.

\section{Controlling molecular individualism}

Molecular individualism is an example of the general phenomenon of transient chaos, ${ }^{53}$ which causes a complex system's dynamics to be unpredictable even though the system eventually achieves a stable fixed point at long time. Here the complex system is the set of coupled Fokker-Planck equations for each "bead" of the polymer ${ }^{54}$ (generally modeled as a bead-spring chain). Controlling molecular individualism then requires adjusting system parameters, i.e. the initial polymer configuration, so that the transient chaos can be stabilized. ${ }^{55}$ For a stretching polymer, one might try to preconfigure the configuration to an adequately unkinked and hence moderately stretched state. Results from previous studies confirm this intuition, because dumbbell-like configurations have been shown to deform uniformly. ${ }^{17}$ Larson's simulations also proved that a sequential shear and then extension can shift the extension distribution to higher stretching. ${ }^{27}$ The UV crosslinked gel serves the same purpose, for it unravels and moderately stretches the DNA molecules as they exit the gel before the hyperbolic contraction (Fig. 5). More generally, any porous structure will serve this purpose, and given the great advancements in microfabrication, it may be more desirable to use a nano-scale microfabricated obstacle course ${ }^{52}$ before the contraction because of the ability to control the obstacle course layout and pore spacing. Attractive studies would be to determine how much the initial configuration distribution must be adjusted to still yield uniform stretching results and how the obstacle course structure can be designed to optimize the stretching efficiency. One may also attempt to tackle these configurational control issues theoretically using non-equilibrium statistical mechanics, however the full chain problem is currently intractable analytically without approximations. ${ }^{54}$

\section{Pseudo-tethered chain model}

In addition to biasing the DNA configurations, the gel aids stretching by slowing down the back of the molecule while the front is leaving, acting as a pseudo-tether (Fig. 7b). Often the back end of the DNA forms a hairpin hook around a piece of the gel ( $c f$. the third and fifth frames of Fig. 6f) which directly provides a tethering force. We can model the DNA as if it is tethered at the gel, but we stress that there is nothing causing the DNA to stick to the gel; it is simply moving at an average lower mobility.

Consequently, we ask what happens to a polymer chain in an ideal, homogeneous extensional field when the position of one of its ends is fixed. Fig. $7 \mathrm{~b}$ shows a schematic of a beadspring polymer tethered at a position $\boldsymbol{x}_{\mathrm{o}}$. The vectors connecting the tethered bead (unfilled) to a bead i (filled) are $\boldsymbol{R}_{\mathrm{i}}$. By tethering the polymer we break the symmetry of the ideal homogeneous extensional field so that the chain stretching will depend on where we tether the molecule. We can easily see this by examining the electrophoretic kinematics in the lab frame. For the tethered chain, the electrophoretic velocity field at each bead $\boldsymbol{V}_{\mathrm{i}}$ is $\boldsymbol{V}_{\mathrm{i}}=\left(\boldsymbol{x}_{\mathrm{o}}+\boldsymbol{R}_{\mathrm{i}}\right) \cdot \nabla(\mu \boldsymbol{E})$, where we now assume $\nabla(\mu \boldsymbol{E})$ is uniform. Breaking this velocity field up in parts, we see that the problem reduces to a superposition of a chain tethered at the origin in an extensional field and a chain tethered in a uniform field (since $\boldsymbol{x}_{\mathrm{o}} \cdot \nabla(\mu \boldsymbol{E})$ is a constant vector equal to $\left.\mu \boldsymbol{E}\left(\boldsymbol{x}_{\mathrm{o}}\right)\right)$. The strength of the uniform stretching part of the field interestingly depends on the coordinates of the DNA leaving the gel. This additional stretching component is low wherever the electrophoretic velocity is low, i.e. near $(\widehat{x}=0, \hat{y}=0)$, however it may dominate the extensional stretching component at large $\hat{y}$ or $\hat{x}$. Furthermore, the ratio of $L / l_{\mathrm{c}}$ controls which of these two mechanisms governs the stretching behavior. In the limit of an abrupt contraction $\left(L / l_{\mathrm{c}} \gg 1\right)$ with a patterned gel at the entrance, the uniform field tethering effect will be the dominating stretching mechanism. In our problem $L / l_{\mathrm{c}}=0.88$ and the gel is located near $=0$ so the dominant stretching mechanism is the pseudo-tethered molecule in the extensional field.

Because we can model the DNA exiting the gel as tethered in the extensional field, it will behave qualitatively like a free chain twice its contour length in an extensional field at steady state. ${ }^{41}$ Since $\tau$ scales as $N^{3 v}$ (or even more strongly considering confinement), the effective $D e$ for a tethered chain in an extensional field is about four times $D e$ for the free chain. Hence, extensions leaving the gel may be larger than the expected steady-state extension for a free chain at a given $D e$. We see evidence of this additional tethering force by looking at the $D e=2$ gel data (Fig. 6b). Note that the stretched molecules contract slightly from $\widehat{x}_{\mathrm{f}}=0.5-1$, meaning that they were initially stretched beyond the steady-state extension at $D e=2\left(\left\langle x_{\mathrm{ex}} / L\right\rangle_{D e}=2\right.$, ss $\left.\sim 0.75\right)$. The same holds at $D e=14$ with the gel, but this chain relaxation is not observable because 
the molecules nearly completely stretch and it takes only $0.02 \tau$ for DNA to traverse the region $0.88<\widehat{x}_{\mathrm{f}}<1.2$.

\section{High fields}

There are a few issues that must be dealt with if device designers seek to use very high field strengths to stretch DNA (or similarly, higher viscosity). First, one must use fields low enough so tensions greater than $\mathrm{O}[100 \mathrm{pN}]$ do not develop and cause the double-stranded DNA to "overstretch"., 9,12,56 This transition value is about an order of magnitude larger than the $\mathrm{O}[1-10 \mathrm{pN}]$ tension in a completely stretched dsDNA. This leaves room to design stretching devices, though researchers must be wary of operating at extremely large electric field strength, as others have observed overstretching in microcontractions operating at high pressure-driven flow rates. ${ }^{12}$ Additionally, high electric fields and velocities can induce nonlinear electrokinetic effects, ${ }^{31-34}$ i.e. forces or flows not directly proportional to $\boldsymbol{E}$. These nonlinear flows have not been studied in conjunction with single DNA electrophoresis, but given previous observations of DNA's polarizability and aggregation, ${ }^{38-40}$ they are bound to arise at high enough fields. Our observations indicate that $D e=23$ may be close to where a nonlinear electrokinetic flow becomes important for T4 DNA. ${ }^{41}$ The strength of this flow would depend on the DNA shape and size, the ionic components of the buffer, and would presumably grow with increasing $\eta$, increasing $\boldsymbol{E}$, and decreasing ionic strength. ${ }^{31-34,57}$

\section{Conclusion}

We have analyzed electrophoretic stretching of DNA, examining the dynamics in electric field gradients of a contraction, at the exit of a porous gel and in the hybrid contraction with a gel at its entrance. We used electric field gradients generated by the channel geometry to stretch DNA. Though our results show that continuous electrophoretic stretching is possible even in a thin slit, they also confirmed the well-known fact that at moderate strain, complete and uniform stretching of DNA in extensional fields is impossible. However, by placing a gel directly before the channel contraction, we preconfigured DNA to moderately extended configurations. Consequently, as the DNA exited the gel and entered the contraction, they strongly and uniformly stretched after only $\varepsilon=4$. The gel additionally supplied a tethering force at the back of the DNA so that at a given $D e$, it is possible to extend DNA greater than the steady state extension of a free molecule. The high stretching uniformity we achieve has not been observed in any other continuous stretching device.

\section{Acknowledgements}

We would like to acknowledge the National Science Foundation CAREER Grant No. CTS-0239012 and US Genomics for funding. We also acknowledge Renaud Falconis and Jean-Louis Viovy for the T4 DNA, Dhananjay Dendukuri and Dan Pregibon for assistance with UV gel experiments, Ju Min Kim for finite element calculations, and
Martin Bazant for discussions of nonlinear electrophoretic effects.

\section{References}

1 D. M. Olive and P. Bean, J. Clin. Microbiol., 1999, 37, 1661.

2 J. O. Tegenfeldt, O. Bakajin, C.-F. Chou, S. S. Chan, R. Austin, W. Fann, L. Liou, E. Chan, T. Duke and E. C. Cox, Phys. Rev. Lett., 2001, 86, 1378.

3 E. Y. Chan, N. M. Goncalves, R. A. Haeusler, A. J. Hatch, J. W. Larson, A. M. Maletta, G. R. Yantz, E. D. Carstea, M. Fuchs, G. G. Wang, S. R. Gullans and R. Gilmanshin, Genome Res., 2004, 14, 1137.

4 D. C. Schwartz, X. Li, L. Hernandez, S. P. Ramnarain, E. J. Huff and Y. K. Wang, Science, 1993, 262, 110.

5 X. Michalet, R. Ekong, F. Fougerousse, S. Rousseaux, C. Schurra, N. Hornigold, M. van Slegtenhorst, J. Wolfe, S. Povey, J. S. Beckmann and A. Bensimon, Science, 1997, 277, 1518.

6 C. A. P. Petit and J. D. Carbeck, Nano Lett., 2003, 3, 1141.

7 E. T. Dimalanta, A. Lim, R. Runnheim, C. Lamers, C. Churas, D. K. Forrest, J. J. de Pablo, M. D. Graham, S. N. Coppersmith, S. Goldstein and D. C. Schwartz, Anal. Chem., 2004, 76, 5293.

8 R. Riehn, M. Lu, Y.-M. Wang, S. F. Lim, E. C. Cox and R. H. Austin, Proc. Natl. Acad. Sci. U. S. A., 2005, 102, 10012.

9 S. B. Smith, Y. Cui and C. Bustamante, Science, 1996, 271, 795.

10 T. T. Perkins, D. E. Smith, R. G. Larson and S. Chu, Science, 1995, 268, 83.

11 S. Ferree and H. W. Blanch, Biophys. J., 2003, 85, 2539.

12 J. W. Larson, G. R. Yantz, R. Charnas, C. M. D'Antoni, M. V. Gallo, K. A. Gillis, L. A. Neely, K. M. Phillips, G. G. Wong, S. R. Gullans and R. Gilmanshin, submitted.

13 J. O. Tegenfeldt, C. Prinz, H. Cao, S. Chou, W. W. Reisner, R. Riehn, Y. M. Wang, E. C. Cox, J. C. Sturm, P. Silberzan and R. H. Austin, Proc. Natl. Acad. Sci. U. S. A., 2004, 101, 10979.

14 P. K. Wong, Y.-K. Lee and C.-M. Ho, J. Fluid Mech., 2003, 497, 55.

15 M. Ueda, J. Biochem. Biophys. Methods, 1999, 41, 153.

16 N. Kaji, M. Ueda and Y. Baba, Appl. Phys. Lett., 2003, 83, 3413.

17 T. T. Perkins, D. E. Smith and S. Chu, Science, 1997, 276, 2016.

18 D. E. Smith and S. Chu, Science, 1998, 281, 1335.

19 D. E. Smith, H. P. Babcock and S. Chu, Science, 1999, 283, 1724.

20 H. P. Babcock, R. E. Teixeira, J. S. Hur, E. S. G. Shaqfeh and S. Chu, Macromolecules, 2003, 36, 4544.

21 J. S. Hur, E. S. G. Shaqfeh, H. P. Babcock and S. Chu, Phys. Rev. E: Stat. Phys., Plasmas, Fluids, Relat. Interdiscip. Top., 2002, 66, 011915.

22 G. C. Randall and P. S. Doyle, Mater. Res. Soc. Symp. Proc., 2004, 790, P3.3.

23 G. C. Randall and P. S. Doyle, Phys. Rev. Lett., 2004, 93, 058102.

24 G. C. Randall and P. S. Doyle, Macromolecules, 2005, 38, 2410.

25 Y.-J. Juang, S. Wang, X. Hu and L. J. Lee, Phys. Rev. Lett., 2004, 93, 268105.

26 P. G. de Gennes, Science, 1997, 276, 1999.

27 R. G. Larson, J. Non-Newtonian Fluid Mech., 2000, 94, 37.

28 R. G. Larson, H. Hu, D. E. Smith and S. Chu, J. Rheol., 1999, 43, 267.

29 B. M. Olivera, P. Baine and N. Davidson, Biopolymers, 1964, 2, 245.

30 D. Long, A. V. Dobrynin, M. Rubinstein and A. Ajdari, J. Chem. Phys., 1998, 108, 1234.

31 S. S. Dukhin, Adv. Colloid Interface Sci., 1993, 44, 1.

32 N. A. Mishchuk and S. S. Dukhin, Electrophoresis, 2002, 23, 2012.

33 M. Z. Bazant, K. Thornton and A. Ajdari, Phys. Rev. E: Stat. Phys., Plasmas, Fluids, Relat. Interdiscip. Top., 2004, 70, 021506.

34 T. M. Squires and M. Z. Bazant, J. Fluid Mech., 2004, 509, 217.

35 C. L. Asbury and G. van den Engh, Biophys. J., 1998, 74, 1024.

36 C. L. Asbury, A. H. Diercks and G. van den Engh, Electrophoresis, 2002, 23, 2658.

37 C.-F. Chou, J. O. Tegenfeldt, O. Bakajin, S. S. Chan, E. C. Cox, N. Darnton, T. Duke and R. H. Austin, Biophys. J., 2002, 83, 2170.

38 H. Isambert, A. Ajdari, J.-L. Viovy and J. Prost, Phys. Rev. Lett., 1997, 78, 971. 
39 H. Isambert, A. Ajdari, J.-L. Viovy and J. Prost, Phys. Rev. E: Stat. Phys., Plasmas, Fluids, Relat. Interdiscip. Top., 1997, 56, 5688.

40 S. Magnusdottir, H. Isambert, C. Heller and J.-L. Viovy, Biopolymers, 1999, 49, 385.

41 G. C. Randall, Single Molecule Analysis of DNA Electrophoresis in Microdevices, $\mathrm{PhD}$ thesis, Massachusetts Institute of Technology, 2006.

42 A. J. Szeri, S. Wiggins and L. G. Leal, J. Fluid Mech., 1991, 228 207.

43 Y. Xia and G. M. Whitesides, Angew. Chem., Int. Ed., 1998, 37, 550.

44 A. Bensimon, A. Simon, A. Chiffaudel, V. Croquette, F. Heslot and D. Bensimon, Science, 1994, 265, 2096.

45 G. C. Randall and P. S. Doyle, Proc. Natl. Acad. Sci. U. S. A., 2005, 102, 10813.

46 J. C. Love, D. B. Wolfe, H. O. Jacobs and G. M. Whitesides, Langmuir, 2001, 17, 6005 .

47 R. G. Larson, Rheol. Acta, 1990, 29, 371.
48 C. Carlsson, A. Larsson, M. Jonsson and B. Nordén, J. Am. Chem. Soc., 1995, 117, 3871 .

49 M. Ueda, H. Ono, Y. Baba, M. Doi and K. Yoshikawa, Biophys. Chem., 1998, 71, 113.

50 P. Mayer, G. W. Slater and G. Drouin, Anal. Chem., 1994, 66, 1777.

51 W. N. Vreeland, R. J. Meagher and A. E. Barron, Anal. Chem., 2002, 74, 4328.

52 S. W. P. Turner, M. Cabodi and H. G. Craighead, Phys. Rev. Lett., 2002, 88, 128103.

53 S. H. Strogatz, Nonlinear Dynamics and Chaos, Addison-Wesley, Reading, MA, 1994.

54 A. N. Gorban and I. V. Karlin, Physica A, 2004, 336, 391

55 Y.-C. Lai, T. Tél and C. Grebogi, Phys. Rev. E: Stat. Phys. Plasmas, Fluids, Relat. Interdiscip. Top., 1993, 48, 709.

56 P. Cluzel, A. LeBrun, C. Heller, R. Lavery, J.-L. Viovy, D. Chatenay and F. Caron, Science, 1996, 271, 792.

57 D. C. Prieve, J. L. Anderson, J. P. Ebel and M. E. Lowell, J. Fluid Mech., 1984, 148, 247.

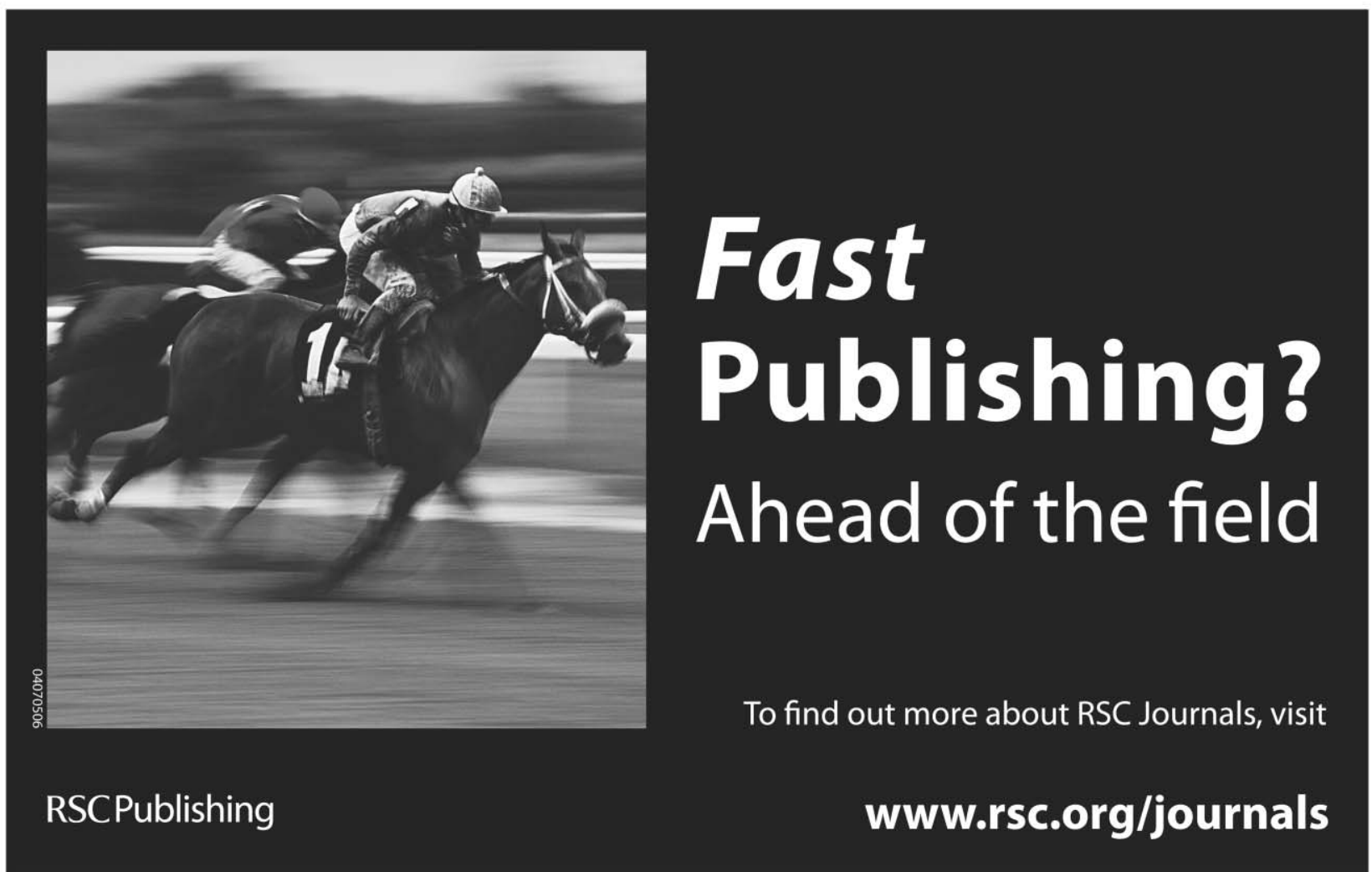

